Cahiers de la recherche sur les droits fondamentaux

Recherche Forles Droits

\title{
Chronique de jurisprudence constitutionnelle française 2010
}

Juliette Lecame, Ellen Lemesle, Ahmed Ouedrogo, Antoine Siffert et Vincent Souty

\section{(2) OpenEdition}

\section{Journals}

Édition électronique

URL : https://journals.openedition.org/crdf/5470

DOI : $10.4000 /$ crdf. 5470

ISSN : 2264-1246

Éditeur

Presses universitaires de Caen

Édition imprimée

Date de publication : 1 décembre 2011

Pagination : 145-154

ISBN : 978-2-84133-396-7

ISSN : 1634-8842

Référence électronique

Juliette Lecame, Ellen Lemesle, Ahmed Ouedrogo, Antoine Siffert et Vincent Souty, «Chronique de jurisprudence constitutionnelle française 2010 ", Cahiers de la recherche sur les droits fondamentaux [En ligne], 9 | 2011, mis en ligne le 01 décembre 2012, consulté le 14 novembre 2022. URL : http:// journals.openedition.org/crdf/5470 ; DOI : https://doi.org/10.4000/crdf.5470 


\section{Chronique de jurisprudence constitutionnelle française $2010^{1}$}

I. Le contrôle formel

A. La compétence du législateur

1. Les incompétences négatives du législateur ordinaire

2. L'encadrement de la compétence du législateur organique

3. Le cas particulier du changement de statut de Mayotte

B. Le contrôle de la légistique

1. Les études d'impact

2. La clarté et la sincérité du débat parlementaire

3. Les réserves d'interprétation

C. L'articulation entre contrôles de conventionnalité et de constitutionnalité

II. Le contrôle substantiel

A. Les principes fondamentaux reconnus par les lois de la République (PFRLR)

B. La garantie des droits devant l'autorité judiciaire

1. L'autorité judiciaire

2. Les principes de droit pénal

C. Le droit de propriété

D. L'organisation du suffrage

1. La clarté et la loyauté du scrutin

2. L'égalité devant le suffrage

Durant l'année 2010, les tables analytiques du Conseil constitutionnel se sont enrichies de vingt-quatre nouvelles décisions - dont neuf portant sur des textes organiques - dans le cadre du contrôle a priori des lois ${ }^{2}$. Force est de constater que les juges de la rue de Montpensier continuent à dérouler leur jurisprudence sur plusieurs points. Aussi, à l'exception d'une censure pour violation du principe d'égalité ${ }^{3}$, se contentent-ils de rappeler régulièrement que des situations différentes peuvent appeler des traitements différents ${ }^{4}$. De même, ils réaffirment

1. Chronique réalisée en collaboration par Juliette Lecame (doctorante contractuelle à l'Université de Caen Basse-Normandie), Ellen Lemesle (doctorante contractuelle à l'Université du Havre), Ahmed Ouedrogo (doctorant à l'Université du Havre), Antoine Siffert (doctorant contractuel à l'Université du Havre) et Vincent Souty (ATER à l'Université du Havre).

2. Étant donné le thème du présent numéro des Cahiers, cette chronique ne s'intéressera qu’à titre accessoire aux questions prioritaires de constitutionnalité rendues sur le fondement de l'article 61-1 de la Constitution.

3. Voir infra.

4. CC, déc. $\mathrm{n}^{\circ}$ 2010-605 DC du 12 mai 2010, Loi relative à l'ouverture à la concurrence et à la régulation du secteur des jeux d'argent et de hasard en ligne (ci-après déc. Jeux d'argent et de hasard), cons. 40; CC, déc. n⿳ 2010-612 DC du 5 août 2010, Loi portant adaptation du droit pénal à l'institution de la Cour pénale internationale, cons. 6; CC, déc. n⿳ 2010-617 DC du 9 novembre 2010, Loi portant réforme des retraites, cons. 13; CC, déc. n 2010-620 DC du 16 décembre 2010, Loi de financement de la Sécurité sociale pour 2011, cons. 14; CC, déc. n 2010-622 DC du 28 décembre 2010, Loi de finances pour 2011, cons. 9. 
l'opportunité qu'a le législateur «de modifier des textes antérieurs ou d'abroger ceux-ci en leur substituant [...] d'autres dispositions, dès lors que, ce faisant, il ne prive pas de garanties légales des exigences constitutionnelles ${ }^{5}$. Ainsi, la réforme de l'aide médicale d'État instaurée par la Loi de finances pour 2011 est conforme au $11^{\mathrm{e}}$ alinéa du préambule de la Constitution de 1946, en ce qu'elle ne troublerait pas l'accès aux soins urgents ainsi que la protection de la santé des mineurs ${ }^{6}$. Enfin, le Conseil fait encore un large usage de sa «balance constitutionnelle» en conciliant - parfois hâtivement - sauvegarde de l'ordre public et différents droits ou libertés individuels, pratique dont la décision $\mathrm{n}^{\circ}$ 2010-613 DC du 7 octobre 2010, Loi interdisant la dissimulation du visage dans l'espace public, est la plus emblématique.

Cela étant, d'autres éléments méritent davantage de développements. En ce qui concerne le contrôle formel (I), le Conseil encadre la compétence du législateur (A) et contrôle la légistique des textes soumis à sa sagacité (B), tout en affinant sa jurisprudence relative à l'articulation entre contrôles de conventionnalité et de constitutionnalité (C). Du point de vue de la protection des droits et libertés (II), il se refuse à dégager de nouveaux principes fondamentaux reconnus par les lois de la République (PFRLR) (A) et se prononce avec prudence sur l'autorité judiciaire et les principes de droit pénal (B), sur le droit de propriété (C) ainsi que sur l'organisation du suffrage (D).

\section{Le contrôle formel}

\section{A. La compétence du législateur}

\section{Les incompétences négatives du législateur ordinaire}

Les requérants ont estimé à plusieurs reprises que le législateur a méconnu l'étendue de sa compétence. Or, à l'exception d'un article de $\mathrm{loi}^{7}$, le Conseil rejette l'ensemble des griefs tirés de l'incompétence négative du législateur. Aussi, par exemple, dans la décision $\mathrm{n}^{0}$ 2010-618 DC du 9 décembre 2010, Loi de réforme des collectivités territoriales, relève-t-il que la création d'une métropole par plusieurs communes et établissements publics de coopération intercommunale ${ }^{8}$ suppose l'intervention du pouvoir réglementaire par décret, que donc les transferts de compétences de plein droit des régions et départements concernés vers les métropoles n'échappent pas au contrôle de l'État ${ }^{9}$. Il est intéressant de constater que, si classiquement le grief de l'incompétence négative est soulevé par des requérants qui reprochent au législateur de laisser trop de liberté au pouvoir réglementaire ${ }^{10}$, il est ici rejeté au motif que justement, l'exécutif n'est pas dépossédé de son pouvoir de décision en matière de création des EPCI - dont les métropoles font parties - et des transferts de compétences correspondants.

En revanche, dans la décision $\mathrm{n}^{\circ}$ 2010-604 DC du 25 février 2010, Loi renforçant la lutte contre les violences de groupes et la protection des personnes chargées d'une mission de service public, le Conseil censure les dispositions qui permettaient aux propriétaires et exploitants d'immeubles collectifs d'habitation d'adresser aux services de police nationale, gendarmerie ou police municipale des images issues de systèmes de vidéosurveillance installés dans les parties communes d'un immeuble. Le Conseil relève que le "législateur est resté en deçà de sa compétence ${ }^{11}$ alors qu'il aurait pu se contenter de relever l'absence de conciliation entre les « exigences constitutionnelles ${ }^{12}$. En retenant le grief de l'incompétence négative, il valide l'intention du législateur qui permet à des personnes privées de transmettre aux forces de l'ordre des images obtenues dans des lieux certes collectifs mais privés et ce, de leur propre initiative.

\section{L'encadrement de la compétence du législateur organique}

Dans la décision $\mathrm{n}^{\circ}$ 2009-597 DC du 21 janvier 2010, Loi organique tendant à permettre à Saint-Barthélemy d'imposer les revenus de source locale des personnes établies depuis moins de cinq ans, le Conseil s'assure de la protection de la compétence du législateur organique. Aussi, d'une part, la loi organique ne peut-elle pas permettre aux collectivités de Saint-Martin et Saint-Barthélemy d'établir avec l'État des conventions leur permettant d'imposer les revenus de source locale des personnes établies depuis moins de cinq ans sans que lesdites conventions soient approuvées ex post par une loi organique ${ }^{13}$. D'autre part, dans une seconde réserve, le Conseil prévient que ces conventions ne pourront avoir pour objet ou pour effet

5. CC, déc. $\mathrm{n}^{\circ}$ 2010-622 DC du 28 décembre 2010, Loi de finances pour 2011 (ci-après Loi de finances), cons. 33. En ce sens, voir également CC, déc. Jeux d'argent et de hasard, cons. 32 sq.; CC, déc. $\mathrm{n}^{0}$ 2010-617 DC du 9 novembre 2010, Loi portant réforme des retraites, cons. 8 sq.

6. CC, déc. Loi de finances, cons. 34 à 37 . Voir T. Gründler, «Aide médicale d'État: de l'incapacité du droit constitutionnel à empêcher la mise en œuvre d'une réforme critiquable», Combats pour les Droits de l'homme, blog.LeMonde.fr, 21 janvier 2011.

7. Voir infra.

8. Ci-après EPCI.

9. CC, déc. $\mathrm{n}^{\circ}$ 2010-618 DC du 9 décembre 2010, Loi de réforme des collectivités territoriales (ci-après déc. Collectivités territoriales), cons. 45 .

10. Ibid., cons. 67

11. CC, déc. $\mathrm{n}^{\circ}$ 2010-604 DC du 25 février 2010, Loi renforçant la lutte contre les violences de groupes et la protection des personnes chargées d'une mission de service public (ci-après déc. Violences de groupes), cons. 20.

12. Ibid., cons. 22. Sur ce point, voir également infra.

13. CC, déc. $\mathrm{n}^{\mathrm{0}}$ 2009-597 DC du 21 janvier 2010, Loi organique tendant à permettre à Saint-Barthélemy d'imposer les revenus de source locale des personnes établies depuis moins de cinq ans, cons. 5; CC, déc. $\mathrm{n}^{\circ}$ 2009-598 DC du 21 janvier 2010, Loi organique modifiant le livre III de la sixième partie du Code général des collectivités territoriales relatif à Saint-Martin, cons. 5. 
de restreindre la compétence du législateur organique en la matière, notamment dans le cas où elles n'aboutiraient pas ${ }^{14}$.

En revanche, le Conseil s'assure que le législateur organique n'aille pas au-delà de ce que prescrit la Constitution. Premièrement, dans la décision $\mathrm{n}^{\circ}$ 2010-608 DC du 24 juin 2010, Loi organique relative au Conseil économique, social et environnemental (CESE), le Conseil censure les dispositions qui prévoyaient l'obligation pour le Gouvernement de remettre un rapport relatif à l'actualisation de la composition du CESE. D'une part, l'article 10 outrepassait le champ de compétence du CESE, et, d'autre part, ses dispositions portaient atteinte aux modalités de fixation de l'ordre du jour des assemblées parlementaires car il exigeait un débat au Parlement. Secondement, par la décision $\mathrm{n}^{\circ}$ 2010-616 DC du 10 novembre 2010, Loi organique relative à la gestion de la dette sociale, le Conseil censure les dispositions élargissant le champ facultatif des lois de financement de la Sécurité sociale (LFSS) à des dispositions «qui ne sont pas directement des dépenses ou des recettes ${ }^{15}$ et qui n'ont que des "incidences ${ }^{16}$ sur l'équilibre financier de la Sécurité sociale. Le Conseil rappelle ainsi que la Constitution limite le champ des LFSS aux « conditions générales de l'équilibre financier ${ }^{17}$. On comprend que le Conseil ne pouvait pas procéder à un simple déclassement étant donné que le contenu de ces lois ne peut être fixé que par des dispositions organiques.

De même, le Conseil censure les dispositions de caractère organique introduites par une loi ordinaire alors qu'il se contente de relever et de déclasser les dispositions ordinaires créées par le législateur organique ${ }^{18}$. Dès lors, par la décision $\mathrm{n}^{\circ}$ 2010-620 DC du 16 décembre 2010, Loi de financement de la Sécurité sociale pour 2011, le Conseil invalide toutes les dispositions portant sur le contenu des lois de financement de la Sécurité sociale au motif que celles-ci doivent résulter d'une loi organique.

\section{Le cas particulier du changement de statut de Mayotte}

Alors que le régime de cette collectivité relevait de la loi organique sur le fondement de l'article 74 de la Constitution, il relèvera de la loi ordinaire sur le fondement de l'article 73 de la Constitution à compter de mars 2011. Aussi le législateur organique doit, tant qu'il en est encore compétent, abroger les dispositions régissant la collectivité de Mayotte. Afin de faciliter le changement de statut, le législateur organique décide de maintenir un régime fiscal dérogatoire au droit commun et caractéristique des collectivités territoriales régies par l'article 74 jusqu'au $1^{\text {er }}$ janvier 2014. Or, ce régime pourra être modifié par le législateur ordinaire «dès la mise en place du département de Mayotte ${ }^{19}$, ce qui semble insatisfaisant du point de vue de l'analyse normative. Si le Conseil justifie cette situation «insolite ${ }^{20}$ par «le caractère non renouvelable ${ }^{21}$ de « cette mesure transitoire ${ }^{22}$, il serait préférable que ce régime dérogatoire fixé par le législateur organique reste en vigueur jusqu'au $1^{\text {er }}$ janvier 2014 - sans que le législateur ordinaire ne puisse le modifier -, et qu'à partir de cette date, le régime fiscal de droit commun, élaboré par le législateur ordinaire sur le fondement de l'article 73 de la Constitution, soit promulgué. Ce qui permettrait d'éviter que le législateur ordinaire vienne défaire ce que le législateur organique a mis en place.

\section{B. Le contrôle de la légistique}

\section{Les études d'impact}

Adopté suite à la réforme de 2008, l'article 8 de la loi organique $\mathrm{n}^{\circ} 2009-403$ du 15 avril 2009, qui complète l'article 39 de la Constitution, prévoit que le Gouvernement doit désormais accompagner ses projets de loi d'une étude d'impact ${ }^{23}$. Mais, comme le souligne justement Anne-Sophie Denolle, leur intérêt semble, en l'état, limité $^{24}$. Le Gouvernement opère un service minimum sur ces études, qui apparaissent parfois, comme c'est le cas pour le projet de Loi interdisant la dissimulation $d u$ visage dans l'espace public, comme un simple exposé des motifs enrichi ${ }^{25}$; et le Conseil n'y porte d'ailleurs pas une attention particulière. Ainsi, dans la décision $\mathrm{n}^{\circ}$ 2010-603 DC du 11 février 2010, Loi organisant la concomitance des renouvellements des conseils généraux et des conseils régionaux, il refuse une censure fondée sur le seul motif qu'une unique étude d'impact a été établie pour différents

14. Ibid.

15. CC, déc. $\mathrm{n}^{\circ}$ 2010-616 DC du 10 novembre 2010, Loi organique relative à la gestion de la dette sociale (ci-après déc. Dette sociale), cons. 9.

16. Ibid.

17. Ibid.

18. Le Conseil opère ainsi trois déclassements: CC, déc. $\mathrm{n}^{\circ}$ 2010-611 DC du 19 juillet 2011, Loi organique relative à l'application de l'article 65 de la Constitution, cons. 6; CC, déc. Dette sociale, cons. 6; CC, déc. $\mathrm{n}^{\circ}$ 2010-619 DC du 2 décembre 2010, Loi organique relative au département de Mayotte, cons. 5 .

19. CC, déc. $\mathrm{n}^{\circ}$ 2010-619 DC du 2 décembre 2010, Loi organique relative au département de Mayotte, cons. 4. Nous soulignons.

20. Commentaire de la décision $\mathrm{n}^{\circ}$ 2010-619 DC du 2 décembre 2010, Les Nouveaux Cahiers du Conseil constitutionnel, cahier $\mathrm{n}^{\circ} 30,2010, \mathrm{p} .11$.

21. CC, déc. $\mathrm{n}^{\circ}$ 2010-619 DC du 2 décembre 2010, Loi organique relative au département de Mayotte, cons. 4.

22. Ibid.

23. L'article 11 de cette loi organique pose les exceptions; les études d'impact ne sont pas exigées en ce qui concerne les projets de révision constitutionnelle, de loi de finances et de financement de la Sécurité sociale, de loi de programmation visés au $21^{\mathrm{e}}$ alinéa de l'article 34 de la Constitution et enfin aux projets de loi prorogeant des états de crise.

24. Voir la contribution d'A.-S. Denolle, au cours de la Journée décentralisée de l'Association française de droit constitutionnel (AFDC), «La révision constitutionnelle de 2008 et l'écriture de la loi», colloque de Caen, 30 novembre 2010, inédit.

25. Ibid. 
projets de $1 \mathrm{ii}^{26}$. En outre, dans la décision $\mathrm{n}^{0}$ 2010-608 DC du 24 juin 2010, Loi organique relative au Conseil économique, social et environnemental, le Conseil renvoie aux réserves émises à l'occasion de la décision n ${ }^{0}$ 2009-579 $\mathrm{DC}^{27}$, dans laquelle il restreint la portée de l'article $8 \mathrm{de}$ la loi organique du point de vue non seulement substantiel mais également procédural. Le Conseil se réserve, en effet, la possibilité de concilier l'exigence du dépôt préalable d'une étude d'impact avec les « exigences de la continuité de la vie de la Nation ${ }^{28}$. Autrement dit, le Conseil ne censurera pas automatiquement une loi au motif que l'étude d'impact obligatoire n'a pas été communiquée aux parlementaires en temps utile.

Dans sa décision Collectivités territoriales, le Conseil relève que les études d'impact ne sont exigées que pour la présentation des projets de loi; or, les dispositions contestées, qui concernaient le mode de scrutin et le nombre des futurs conseillers territoriaux ainsi que la répartition des compétences entre les collectivités territoriales, ont été introduites par voie d'amendement parlementaire ${ }^{29}$. Le Gouvernement pourrait, le cas échéant, contourner l'obligation d'effectuer une telle étude en demandant à des parlementaires de déposer une proposition de loi ou, simplement, des amendements.

\section{La clarté et la sincérité du débat parlementaire}

Le Conseil étoffe sa jurisprudence relative à la clarté et à la sincérité, mais sans parvenir à clarifier différents points ${ }^{30}$. La qualification d' «exigence constitutionnelle» est retenue pour cinq décisions ${ }^{31}$, mais pas dans la rédaction de la décision Loi de finances qui omet le terme d' «exigence ${ }^{32}$. Malgré ces contorsions sémantiques, le Conseil continue néanmoins de se référer à la clarté et à la sincérité pour justifier l'encadrement de la procédure d'adoption des normes législatives. Dans la décision n ${ }^{\circ}$ 2010-603 DC, il rejette l'incompatibilité entre la procédure du vote bloqué prévue à l'article 44, alinéa 3, de la Constitution et les exigences de clarté et de sincérité ${ }^{33}$, ce vote bloqué n'empêchant pas la discussion de chacune des dispositions du texte en question ${ }^{34}$.

En revanche, l'articulation des exigences de clarté et de sincérité du(es) débat(s) parlementaire(s) avec la prohibition des cavaliers reste assez obscure ${ }^{35}$. En témoigne la décision $E I R L$, dans laquelle le Conseil censure des dispositions n'ayant pas de lien direct avec celles qui figurent dans le projet de loi présenté, ajoutant au passage que ces dispositions «ont été adoptées en méconnaissance de la clarté et de la sincérité du débat parlementaire ${ }^{36}$, pour finir par fonder sa décision sur l'article 45 de la Constitution. Mais cet article modifié en 2008 conditionne la recevabilité des amendements à l'existence d'un lien, au moins indirect, avec le texte déposé. On peut dès lors se poser la question de l'utilité de la référence à la clarté et à la sincérité du débat dans cette décision, d'autant plus que dans la décision $\mathrm{n}^{\circ}$ 2010-617 DC du 9 novembre 2010, Loi portant réforme des retraites, seul l'article 45 est mentionné pour justifier l'annulation d'un cavalier issu d'un amendement ${ }^{37}$. De même, les censures des cavaliers relevés dans la loi de financement de la Sécurité sociale ${ }^{38}$ ou dans les lois de finances et de finances rectificatives ${ }^{39}$ s'opèrent sans référence aucune à l'exigence de clarté et de sincérité des débats.

\section{Les réserves d'interprétation}

En permettant de valider une loi tout en en corrigeant les lacunes, la réserve d'interprétation reste l'instrument privilégié du Conseil constitutionnel. Réserve directive, comme au considérant 5 de la décision Dette sociale, lorsque le Conseil intime au législateur organique de respecter «l'équilibre financier de la Sécurité sociale de l'année à venir» en interdisant le transfert sans compensation de recettes affectées à la Sécurité sociale au profit de la Caisse d'amortissement de la dette sociale. Réserve constructive, lorsqu'il précise dans sa décision EIRL de juin 2010, que la soustraction par le débiteur de certains biens affectés d'un gage ne porte pas atteinte aux conditions d'exercice du

26. CC, déc. $\mathrm{n}^{\circ}$ 2010-603 DC du 11 février 2010, Loi organisant la concomitance des renouvellements des conseils généraux et des conseils régionaux (ci-après déc. Renouvellement des conseils généraux et régionaux), cons. 3 et 4 .

27. CC, déc. $\mathrm{n}^{\circ}$ 2010-608 DC du 24 juin 2010, Loi organique relative au Conseil économique, social et environnemental, cons. 12.

28. CC, déc. $\mathrm{n}^{\circ}$ 2009-579 DC du 9 avril 2009, Loi organique relative à l'application des articles 34-1, 39 et 44 de la Constitution, cons. 17.

29. CC, déc. Collectivités territoriales, cons. 8

30. Voir, sur ce point, l'article de G. Lebreton, «La sincérité des débats parlementaires», in La Sincérité en droit, O. Le Bot (dir.), Bruxelles, Larcier, 2011, p. 169-186.

31. CC, déc. Renouvellement des conseils généraux et régionaux; CC, déc. Jeux d'argent et de hasard; CC, déc. $\mathrm{n}^{\circ} 2010-607$ DC du 10 juin 2010, Loi relative à l'entrepreneur individuel à responsabilité limitée (ci-après déc. EIRL); CC, déc. n² 2010-617 DC du 9 novembre 2010 , Loi portant réforme des retraites; $\mathrm{CC}$, déc. Collectivités territoriales.

32. De même, le Conseil se réfère alternativement «aux débats parlementaires» (CC, déc. Renouvellement des conseils généraux et régionaux, cons. 8 ; CC, déc. Jeux d'argent et de hasard, cons. 3; CC, déc. Collectivités territoriales, cons. 9; CC, déc. Loi de finances, cons. 5) ou "au débat» au singulier (CC, déc. EIRL, cons. 6; CC, déc. n 2010-617 DC du 9 novembre 2010, Loi portant réforme des retraites, cons. 2).

33. CC, déc. Renouvellement des conseils généraux et régionaux, cons. 8 et 9.

34. Voir en ce sens CC, déc. $\mathrm{n}^{\circ}$ 59-5 DC du 15 janvier 1960, Résolution modifiant les articles 95 et 96 du règlement de l'Assemblée nationale, cons. 1.

35. Voir, par exemple, CC, déc. $\mathrm{n}^{\circ}$ 2009-578 DC du 18 mars 2009, Loi de mobilisation pour le logement et la lutte contre l'exclusion, cons. 30.

36. CC, déc. EIRL, cons. 6

37. CC, déc. $\mathrm{n}^{\circ}$ 2010-617 DC du 9 novembre 2010, Loi portant réforme des retraites, cons. 24 .

38. CC, déc. $\mathrm{n}^{\circ}$ 2010-620 DC du 16 décembre 2010, Loi de financement de la Sécurité sociale pour 2011, cons. 16 sq.

39. CC, déc. Loi de finances, cons. 55 ; CC, déc. $\mathrm{n}^{\circ}$ 2010-623 DC du 28 décembre 2010, Loi de finances rectificative pour 2010 , cons. 6. 
droit de propriété des créanciers, à la condition expresse que ceux-ci soient effectivement mis à même de s'y opposer. Enfin, réserve neutralisante pour exclure une interprétation litigieuse d'une loi ou pour évacuer une disposition inconstitutionnelle.

Sur ce dernier point, la réserve contenue dans la décision relative à la Loi interdisant la dissimulation du visage dans l'espace public est éloquente. «L'interdiction de dissimuler son visage dans l'espace public ne saurait, sans porter une atteinte excessive à l'article 10 de la Déclaration de 1789, restreindre l'exercice de la liberté religieuse dans les lieux de culte ouverts au public ${ }^{40}$. En focalisant sa décision sur cette inconstitutionnalité manifeste, le Conseil évite de s'attarder sur la question centrale de la liberté personnelle en n'opérant qu'un bref et classique contrôle sur l'absence de disproportion manifeste, pourtant contestable, entre la sauvegarde de l'ordre public et la garantie des droits constitutionnellement protégés. En l'espèce, l'intérêt de la réserve semble double: garantir le respect de la liberté religieuse, mais surtout délivrer rapidement un brevet de constitutionnalité de la loi tout en affichant un ersatz de censure.

Conçues initialement pour encadrer le travail législatif, les réserves deviennent ici un moyen de le valider. Cette légitimation se retrouve aux considérants 12 et 32 de la décision Violences de groupes. Le nouvel article 222-14-2 du Code pénal qui réprime le fait de participer sciemment à un groupement en vue de commettre des violences ou des dommages est jugé conforme au principe de responsabilité du fait personnel, «à la condition que la préparation de ces infractions soit caractérisée par un ou plusieurs faits matériels accomplis par l'auteur lui-même ou connus de lui ${ }^{41}$. Faisant sienne l'interprétation du législateur, le Conseil valide également la circonstance aggravante qui sanctionne l'intrusion non autorisée dans un établissement scolaire commise en réunion par une personne porteuse d'une arme, puisque cette peine aggravée ne sera applicable qu'à cette seule personne. En explicitant la portée exacte de ces dispositions et en réfutant les critiques de l'opposition parlementaire, le juge constitutionnel opère une interprétation «que l'on pourra qualifier de neutralisante ${ }^{42}$.

\section{L'articulation entre contrôles de conventionnalité et de constitutionnalité}

Ainsi qu'il le rappelle fréquemment depuis sa décision $I V G$ de $1975^{43}$, le Conseil constitutionnel n'est pas le juge de la conventionnalité des lois. En 2010, il réaffirme cette position à deux reprises dans le cadre du contrôle a prior ${ }^{44}$, en reprenant les termes mêmes de sa jurisprudence de 1975. Il adopte la même démarche dans le cadre de la QPC ${ }^{45}$.

Malgré l'apparente banalité de ces rappels, le Conseil constitutionnel se prononce cette année pourtant dans un nouveau contexte: la mise en place de la procédure de contrôle de constitutionnalité a posteriori. Or, déjà soulevée par la doctrine, la question de la compatibilité de ce mécanisme au droit communautaire ${ }^{46}$ pousse la Cour de cassation à saisir la Cour de justice de l'Union européenne (CJUE) d'une question préjudicielle à ce sujet dans un arrêt du 16 avril $2010^{47}$, au risque de s'exposer à de sérieuses critiques ${ }^{48}$ et d'être accusée de «chercher à faire obstacle à l'application du mécanisme de la QPC» ${ }^{49}$. Alors que la solution européenne n'est pas encore connue, le Conseil constitutionnel est saisi pour se prononcer sur la conformité à la Constitution de la Loi relative à l'ouverture à la concurrence en ligne et à la régulation du secteur des jeux d'argent et de hasard en ligne ${ }^{50}$. Il aurait pu se contenter de rejeter les griefs d'inconventionnalité soulevés par les requérants, mais il saisit l'occasion pour répondre aux craintes de la Cour de cassation en soulignant trois points importants, qui tendent à confirmer la conformité du mécanisme de la QPC, à savoir que ce mécanisme:

- «ne limite pas la compétence des juridictions administratives et judiciaires pour faire prévaloir ces engagements [européens] sur une disposition législative incompatible avec eux ${ }^{51}$;

- n'empêche pas le juge de «prendre toutes les mesures provisoires ou conservatoires nécessaires ${ }^{52}$;

40. CC, déc. $\mathrm{n}^{\circ}$ 2010-613 DC du 7 octobre 2010, Loi interdisant la dissimulation du visage dans l'espace public, cons. 5 .

41. CC, déc. Violences de groupes, cons. 12

42. H. Lessafre, «La décision du Conseil constitutionnel sur la Loi renforçant la lutte contre les violences de groupes et la protection des personnes chargées d'une mission de service public : un épilogue hâtif», Petites Affiches, $\mathrm{n}^{\circ}$ 46, 5 mars 2010, p. 3 .

43. CC, déc. $\mathrm{n}^{\circ}$ 74-54 DC du 15 janvier 1975, Loi relative à l'interruption volontaire de grossesse, cons. 7 .

44. CC, déc. Jeux d'argent et de hasard, cons. 11 et 12 ; CC, déc. ${ }^{\circ}$ 2010-612 DC du 5 août 2010, Loi portant adaptation du droit pénal à l'institution de la Cour pénale internationale (ci-après déc. CPI), cons. 5.

45. CC, déc. $\mathrm{n}^{\circ}$ 2010-4/17 QPC du 22 juillet 2010, M. Alain C. et autres [Indemnité temporaire de retraite outre-mer], cons. 11: «il n'appartient pas au Conseil constitutionnel, saisi en application de l'article 61-1 de la Constitution, d'examiner la compatibilité des dispositions contestées avec les engagements internationaux de la France; que l'examen d'un tel grief relève de la compétence des juridictions administratives et judiciaires ».

46. L. Burgorgue-Larsen, «Question préjudicielle de constitutionnalité et contrôle de conventionnalité. État des lieux de leurs liaisons (éventuellement dangereuses) sur la base du projet de loi organique appliquant l'article $61 \$ 1$ de la Constitution", Revue française de droit administratif, $\mathrm{n}^{\circ} 4,2009$, p. 787

47. Cour cass., 16 avril 2010, $\mathrm{n}^{\circ}$ 10-40.001 [QPC], AJDA, 2010, p. 1022-1029, note P. Manin.

48. Sur ce point, voir G. Carcassonne et N. Molfessis, «La Cour de cassation à l'assaut de la question prioritaire de constitutionnalité», Le Monde, 22 avril 2010; A. Levade, «Renvoi préjudiciel versus question prioritaire de constitutionnalité: la Cour de cassation cherche le conflit! ", Dalloz, 2010, chron. 1254

49. P. Manin, «La question prioritaire de constitutionnalité et le droit de l’Union européenne», AJDA, 2010, p. 2188.

50. CC, déc. Jeux d'argent et de hasard.

51. Ibid., cons. 13

52. Ibid., cons. 14 
- «ne [prive] pas davantage les juridictions administratives et judiciaires, [...] de la faculté [...] de saisir la Cour de justice de l'Union européenne d'une question préjudicielle ${ }^{53}$.

Cette intervention du Conseil constitutionnel a permis de participer au tant désiré « dialogue des juges " ${ }^{54}$. La CJUE, dans sa réponse à la Cour de cassation ${ }^{55}$, reprend d'ailleurs, à peu de chose près, la conception du juge constitutionnel. Ce dernier élude toutefois avec soin la question de l'examen par priorité de la question de constitutionnalité sur la question de conventionnalité. En effet, l'article 23-2, alinéa 5, de l'ordonnance du 7 novembre $1958^{56}$ prévoit qu' «en tout état de cause, la juridiction doit, lorsqu'elle est saisie de moyens contestant la conformité d'une disposition législative, d'une part, aux droits et libertés garantis par la Constitution et, d'autre part, aux engagements internationaux de la France, se prononcer par priorité sur la transmission de la question de constitutionnalité au Conseil d'État ou à la Cour de cassation ». Or, selon la CJUE, la QPC est conforme au droit communautaire dès lors que les juridictions nationales restent libres de la saisir, «à tout moment de la procédure qu'elles jugent approprié», d'une question préjudicielle. La précision "à tout moment de la procédure» tend à remettre en cause le caractère prioritaire de la QPC (point sur lequel le Conseil était resté silencieux), dès l'instant où les juges estiment approprié de saisir la CJUE d'une question préjudicielle avant de transmettre une question de constitutionnalité ${ }^{57}$.

En outre, toujours à propos du droit communautaire, le Conseil constitutionnel précise dans sa décision du 12 mai 2010 que le respect de l'exigence constitutionnelle de transposition des directives ne peut être invoqué dans le cadre de la QPC car elle ne relève pas des «droits et libertés que la Constitution garantit ${ }^{58}$, condition mentionnée à l'article 61-1. Le Conseil rappelle les limites du contrôle de cette exigence, notamment le fait que la transposition de la directive ne saurait aller à l'encontre d'une règle ou d'un principe inhérent à l'identité constitutionnelle de la France ${ }^{59}$. Se pose alors la question de savoir si l'atteinte à un tel principe peut être invoquée dans le cadre de la QPC. Ce n'est pas impossible, au vu de la décision $\mathrm{n}^{\circ}$ 2010-79 QPC du 17 décembre 2010,
M. Kamel D. ${ }^{60}$, dans laquelle le Conseil refuse d'examiner la question prioritaire de constitutionnalité car les dispositions contestées ne mettaient en cause aucune règle ni aucun principe inhérent à l'identité constitutionnelle de la France ${ }^{61}$. Enfin, le Conseil refuse d'étendre le raisonnement qu'il tient pour les directives européennes au statut de la Cour pénale internationale, contrairement aux attentes des auteurs de la saisine. Il n'existe ainsi aucune exigence d'intégration en droit interne des règles relatives à cet accord international ${ }^{62}$.

\section{Le contrôle substantiel}

\section{A. Les principes fondamentaux reconnus par les lois de la République (PFRLR)}

Durant la période étudiée, le Conseil confirme sa jurisprudence en matière de principes fondamentaux reconnus par les lois de la République et refuse de suivre les invitations faites par les requérants pour en dégager de nouveaux. Dans la décision Jeux d'argent et de hasard, il refuse de reconnaître le principe de leur prohibition comme un PFRLR ${ }^{63}$. Fidèle à sa conception classique, il constate que s'il existe une série de lois républicaines antérieures à 1946 qui prohibent les jeux d'argent et de hasard, celles-ci ont toujours été assorties de possibilités de dérogations ${ }^{64}$ : l'interdiction n'ayant jamais été absolue, le Conseil ne l'érige pas en PFRLR ${ }^{65}$. Dans la décision Collectivités territoriales, il réfute le qualificatif à la clause «de compétence générale des collectivités territoriales». Celle-ci, prévue aux articles L. 2121-29, L. 3211-1 et L. 4221-1 du Code général des collectivités territoriales (CGCT), permet aux collectivités d'agir dans tout domaine présentant un intérêt pour la collectivité, même en l'absence de texte, dans la limite des compétences dévolues à d'autres personnes publiques. L'article 73 de la loi déférée vient limiter la portée de cette clause pour les départements et les régions. Invoquant la portée de l'article $48 \mathrm{de}$ la loi du 10 août 1871 qui confère cette compétence générale aux départements ${ }^{66}$, le Conseil, sibyllin, se borne à constater qu'elle ne présente pas un caractère de généralité suffisant pour lui conférer la qualité de PFRLR ${ }^{67}$.

53. CC, déc. Jeux d'argent et de hasard, cons. 15.

54. H. Labayle, "Question prioritaire de constitutionnalité et question préjudicielle: ordonner le dialogue des juges? », RFDA, 2010, p. 659.

55. CJUE, 22 juin 2010, affaire C-188/10, AJDA, 2010, p. 1578, chron. E. Broussy, M. Aubert et F. Donnat.

56. Article 23-2, alinéa 5, de l'ordonnance $\mathrm{n}^{\circ}$ 58-1067 du 7 novembre 1958 portant loi organique sur le Conseil constitutionnel.

57. Voir P. Manin, «La question prioritaire de constitutionnalité et le droit de l'Union européenne, un bilan provisoire», AJDA, 2010, p. 2188.

58. CC, déc. Jeux d'argent et de hasard, cons. 19.

59. Principe dégagé dans la décision $\mathrm{n}^{\circ}$ 2004-496 DC du 10 juin 2004, Loi pour la confiance dans l'économie numérique, cons. 7.

60. CC, déc. $\mathrm{n}^{\circ}$ 2010-79 QPC du 17 décembre 2010, M. Kamel D. [Transposition d'une directive].

61. Ibid., cons. 4 .

62. CC, déc. $C P I$, cons. 5 .

63. CC, déc. Jeux d'argent et de hasard, cons. 7

64. Le Conseil fait ainsi référence à l'article 136 de la loi de finances du 31 mai 1933 créant la Loterie nationale ainsi qu'à la loi du 15 juin 1907 réglementant le jeu dans les cercles et les casinos des stations balnéaires, thermales et climatériques.

65. Le Conseil avait procédé de la même manière dans sa décision $\mathrm{n}^{\circ}$ 88-244 DC du 20 juillet 1988, Loi portant amnistie, cons. 12.

66. Article partiellement reproduit par le Conseil dans le cons. 54 de sa décision.

67. CC, déc. Collectivités territoriales, cons. 54. 


\section{B. La garantie des droits devant l'autorité judiciaire}

\section{L'autorité judiciaire}

La notion même d'autorité judiciaire est susceptible de controverses. Selon la Cour européenne des Droits de l'homme, les magistrats du parquet ne sont pas des autorités judiciaires en raison de leur manque d'indépendance à l'égard du pouvoir exécutif ${ }^{68}$. Le Conseil affirme, pour sa part, dans sa décision sur la garde à vue que «l'autorité judiciaire comprend à la fois les magistrats du siège et du parquet ${ }^{69}$. Ainsi, du point de vue du juge constitutionnel, les magistrats du parquet, bien que placés sous l'autorité du garde des Sceaux, sont des autorités judiciaires. Le Conseil affirme toutefois à deux reprises que la décision du ministère public doit être susceptible de recours sous peine de contredire le droit à un recours effectif, notamment dans sa décision $\mathrm{n}^{0} 2010-614$ DC du 4 novembre $2010^{70}$. En l'espèce, la loi soumise au Conseil prévoyait que le ministère public puisse mettre à exécution une décision de raccompagnement d'un mineur roumain dans son pays d'origine sans que ne soit saisi le juge des enfants. Le Conseil estime que l'absence de recours contre cette décision est contraire au droit à un recours effectif sur le fondement de l'article 16 de la Déclaration universelle des Droits de l'homme et du citoyen (DDHC). Or, si l'on met en parallèle la décision sur la garde à vue, la jurisprudence du Conseil constitutionnel peut sembler paradoxale. D'un côté, le juge affirme que le droit à un recours effectif est violé parce que seul le parquet intervient dans la procédure, mais de l'autre, il estime que les magistrats du parquet sont des autorités judiciaires et peuvent décider du placement et du maintien en garde à vue sans que ces décisions ne soient susceptibles de recours. Le Conseil aura l'occasion de se prononcer à nouveau sur la garde à vue lorsque la réforme sera adoptée, et il sera intéressant de voir si sa position a évolué.

Le Conseil est également amené à se prononcer sur l'indépendance de l'autorité judiciaire dans sa décision $\mathrm{n}^{\circ}$ 2010-611 DC du 19 juillet 2010, Loi organique relative à l'application de l'article 65 concernant la réforme du Conseil supérieur de la magistrature (CSM). Il précise alors, en s'appuyant sur l'article 16 de la Déclaration de 1789 , l'article 64 et le nouvel article 65 de la Constitution, que l'indépendance du CSM concourt à l'indépendance de l'autorité judiciaire ${ }^{71}$. Il veille à son respect en adoptant ainsi deux réserves d'interprétation ${ }^{72}$. En outre, la réforme introduit la possibilité pour les justiciables de saisir le CSM et de déposer une plainte à l'encontre d'un magistrat qui demeure saisi de la procédure ayant occasionné cette plainte. Toutefois, le Conseil estime qu'en ne fixant comme seuls critères de recevabilité de la requête que la nature de la procédure et la gravité du manquement, le législateur n'a pas adopté les garanties appropriées pour que la mise en œuvre d'une procédure disciplinaire à l'initiative d'un justiciable «ne porte pas atteinte à l'impartialité des magistrats mis en cause ou à leur indépendance à l'égard des parties à la procédure et ne méconnaisse pas l'objectif de valeur constitutionnelle de bonne administration de la justice ${ }^{73}$. L'indépendance du CSM ne lui permet toutefois pas de bénéficier de l'autonomie budgétaire garantie par le Conseil au titre de la séparation des pouvoirs ${ }^{74}$.

Concernant le rôle de l'autorité judiciaire, le Conseil affirme, dans la décision CPI, que l'exigence constitutionnelle de recherche des auteurs d'infractions n'implique pas la reconnaissance de la compétence des juridictions françaises «à l'égard de crimes commis à l'étranger sur une victime étrangère et dont l'auteur, de nationalité étrangère, se trouve en France ${ }^{75}$. Ainsi, sur le fondement de la complémentarité entre la CPI et les juridictions françaises, il estime qu'il revient au législateur de déterminer les conditions dans lesquelles les mécanismes de la compétence universelle et de la territorialité peuvent être enclenchés et appliqués ${ }^{76}$. Enfin, dans la même décision, le Conseil distingue les crimes contre l'humanité des crimes de guerre - prévus aux articles 7 et 8 du Statut de Rome du 17 juillet 1998 -, contrairement aux requérants qui estimaient ces infractions de nature similaire et attendaient que le législateur les rende toutes deux «imprescriptibles» au titre du principe d'égalité devant la loi pénale ${ }^{77}$.

68. CEDH, 23 novembre 2010, $\mathrm{n}^{\circ} 37104 / 06$, France Moulin c. France, $₫ 57$.

69. CC, déc. $\mathrm{n}^{\circ}$ 2010-14/22 QPC du 30 juillet 2010, [garde à vue], cons. 26.

70. CC, déc. $\mathrm{n}^{\circ}$ 2010-614 DC du 4 novembre 2010, Loi autorisant l'approbation de l'accord entre le Gouvernement de la République française et le Gouvernement de la Roumanie relatif à une coopération en vue de la protection des mineurs roumains isolés sur le territoire de la République française et à leur retour dans leur pays d'origine ainsi qu'à la lutte contre les réseaux d'exploitation concernant les mineurs, cons. 5 ; CC, déc. $\mathrm{n}^{\circ}{ }_{2010-}$ 38 QPC du 29 septembre 2010, M. Jean-Yves G. [Amende forfaitaire et droit au recours], cons. 7.

71. CC, déc. $\mathrm{n}^{\circ}$ 2010-611 DC du 19 juillet 2010, Loi organique relative à l'application de l'article 65, cons. 5.

72. Il s'oppose ainsi à ce que le premier président et le procureur de la Cour de cassation "participent aux décisions ou aux avis relatifs aux magistrats qui ont, antérieurement, été membres du Conseil supérieur de la magistrature sous leur présidence» (cons. 12).

73. Ibid., cons. 21 et 22

74. Ibid., cons. 13

75. CC, déc. $C P I$, cons. 12 .

76. À cet égard, on peut d'ailleurs relever qu'il vient restreindre la portée de l'objectif à valeur constitutionnelle en limitant celle-ci au champ de compétence territoriale ou personnelle. Au-delà, on peut se poser la question d'une éventuelle compatibilité entre les mécanismes retenus en droit français pour intégrer l'idée de compétence universelle et la façon dont celle-ci est conçue en droit international.

77. La prescription des crimes de guerre a été portée à 30 ans. Seuls les crimes contre l'humanité sont imprescriptibles. Relevant que ces infractions sont de «nature différente», le Conseil estime que la différenciation introduite par l'article 7 de la Loi portant adaptation du droit pénal à l'institution de la Cour pénale internationale est justifiée et ne viole pas l'article 6 de la Déclaration des Droits de l'homme et du citoyen (cons. 6 et 7 ). 


\section{Les principes de droit pénal}

Le principe de légalité des délits et des peines est une "garantie fondamentale des droits de la personne devant les juridictions répressives ${ }^{78}$. Le Conseil se réfère à ce principe dans sa décision Violences de groupes, décision dans laquelle il insiste sur la nécessité de concilier l'exigence de sauvegarde de l'ordre public et le respect des libertés individuelles ${ }^{79}$. Une telle exigence est fondée sur les articles 7 et 8 de la DDHC. Selon ce principe, le législateur est tenu d'élaborer «des dispositions suffisamment précises et des formules non équivoques ${ }^{80}$ lorsqu'il définit les délits et les peines. Le principe de légalité peut, de ce fait, se rattacher à l'objectif de valeur constitutionnelle d'accessibilité et d'intelligibilité de la loi en vue de protéger les citoyens contre les interprétations contraires à la Constitution ${ }^{81}$.

Dans la décision précitée du 25 février 2010, les juges estiment notamment que le législateur, dans la définition de nouvelles infractions telles que le fait de participer «sciemment» à un groupement en vue de préparer des actes de violence contre des personnes ou en vue de s'en prendre à des constructions, de dégrader des biens, de pénétrer dans des établissements scolaires, n'a pas employé de formules «obscur[e]s» ni «ambigu[ë]s» ${ }^{82}$. Tout comme dans sa décision $\mathrm{n}^{\circ}$ 2010-492 DC du 2 mars $2004^{83}$, le Conseil affine son analyse à propos des éléments matériels de l'infraction. Il faut lire en filigrane que les juges exhortent le législateur à élucider davantage la notion de «groupement», à légiférer tout en tenant compte des motivations de chaque individu à rejoindre un "groupe», afin de faciliter la recherche des auteurs d'infractions.

Outre l'exigence constitutionnelle de précision de la loi pénale, les juges constitutionnels se penchent sur la transmission aux forces de l'ordre d'images provenant de la vidéosurveillance dans les «parties communes d'un immeuble à usage d'habitation ». Ainsi, conformément à la décision dite Hadopi ${ }^{84}$, le Conseil réaffirme qu'il appartient au législateur de trouver l'équilibre entre deux exigences constitutionnelles potentiellement contradictoires. En l'espèce, les juges estiment que le législateur méconnaît l'article 34 de la Constitution en autorisant la transmission d'images issues de la vidéosurveillance «des parties communes d'un immeuble à usage d'habitation " aux forces de l'ordre (police nationale, police municipale, gendarmerie). Pour ainsi dire, il ne faut pas privilégier la recherche des auteurs d'une infraction au détriment du respect de la vie privée ${ }^{85}$.

\section{Le droit de propriété}

En 2010, la diversité des décisions du juge constitutionnel relatives au droit de propriété, tant privée que publique, permet de refléter le caractère " protéiforme ${ }^{86}$ dudit droit.

En faisant bénéficier les atteintes au droit des créanciers du champ de protection des conditions d'exercice du droit de propriété, le Conseil opère, dans sa décision EIRL, une «modernisation du droit constitutionnel de propriété ${ }^{87}$. S'il valide la possibilité pour un entrepreneur individuel à responsabilité limitée de séparer patrimoine personnel et patrimoine professionnel et d'opposer cette déclaration d'affectation de biens "aux créanciers dont les droits sont nés antérieurement à son dépôt », le Conseil formule néanmoins une réserve d'interprétation constructive afin de garantir l'effectivité des articles 2 et 4 de la DDHC. La déclaration d'affectation n'est opposable qu'à la condition que les créanciers en «soient personnellement informés [ainsi que] de leur droit de former opposition ${ }^{88}$. Le Conseil ne se réfère pas à l'article 17 de la Déclaration française de 1789 , ce qui signifie que l'État n'est pas tenu d'une quelconque obligation de pallier la défaillance fortuite de l'entrepreneur individuel. À l'inverse, le législateur doit désormais veiller à ne pas attenter aux conditions d'exercice du droit de propriété des créanciers en permettant à un débiteur d'organiser son insolvabilité.

Concernant la propriété publique, le juge constitutionnel se limite, en revanche, à sa jurisprudence antérieure. Au considérant 44 de sa décision Collectivités territoriales ${ }^{89}$, il rappelle que «le droit au respect des biens garanti [par les articles 6 et 13 de la DDHC ainsi que 2

78. CC, déc. Violences de groupes, cons. 4.

79. Depuis sa décision $n^{\circ} 89-261$ DC du 28 juillet 1989, Loi relative aux conditions de séjour et d'entrée des étrangers en France (cons. 12), le Conseil reconnaît expressément la sauvegarde de l'ordre public comme objectif de valeur constitutionnelle.

80. CC, déc. $\mathrm{n}^{\circ}$ 2003-475 DC du 24 juillet 2003, Loi portant réforme de l'élection des sénateurs, cons. 20 ; CC, déc. $\mathrm{n}^{\circ} 2004-500$ DC du 29 juillet 2004 , Loi organique relative à l'autonomie financière des collectivités territoriales, cons. 13.

81. Sur ce point, voir L. Baghestani, «Le principe de légalité des délits et des peines», Petites Affiches, $\mathrm{n}^{\circ} 8,12$ janvier 2001, p. 8.

82. CC, déc. Violences de groupes, cons. 12.

83. CC, déc. $\mathrm{n}^{\circ}$ 2004-492 DC du 2 mars 2004, Loi portant adaptation de la justice aux évolutions de la criminalité, cons. 14.

84. CC, déc. $\mathrm{n}^{\circ}$ 2009-580 DC du 10 juin 2009, Loi favorisant la diffusion et la protection de la création sur Internet, cons. 23.

85. CC, déc. Violences de groupes, cons. 22 et 23.

86. H. Pauliat, «Le droit de propriété devant le Conseil constitutionnel et la Cour européenne des Droits de l'homme», Revue de droit public, 1995, p. 1446.

87. S. Mouton, «La modernisation du droit constitutionnel de propriété: à propos de la constitutionnalisation du droit de propriété des créanciers », Recueil Dalloz, ${ }^{\circ}$ 38, 4 novembre 2010, p. 2553-2557.

88. CC, déc. EIRL, cons. 9 .

89. Voir également CC, déc. $\mathrm{n}^{\circ}$ 2009-594 DC du 3 décembre 2009, Loi relative à l'organisation et à la régulation des transports ferroviaires et portant diverses dispositions relatives aux transports, cons. 15 . 
et 17] ne s'oppose pas à ce que le législateur procède au transfert gratuit de dépendances du domaine public entre personnes publiques» - en l'espèce, entre des collectivités territoriales et une métropole au sens de l'article L. 5217-1 du CGCT. C'est avec la même concision que, dans sa décision $\mathrm{n}^{\circ}$ 2010-601 DC du 4 février 2010, Loi relative à l'entreprise publique La Poste et aux activités postales, le Conseil valide la transformation de La Poste - jusqu'alors établissement public à caractère industriel et commercial - en société anonyme. Pour rejeter le grief fondé sur la violation de l'alinéa 9 du préambule de la Constitution de 1946, il juge que l'article $1^{\text {er }}$ de la loi déférée «n'a ni pour objet ni pour effet de transférer La Poste au secteur privé ${ }^{90}$, notamment puisque l'État reste actionnaire majoritaire du capital. Le Conseil rejette ainsi les prétentions des requérants qui souhaitaient voir constitutionnalisé le caractère de service public national de La Poste, afin d'éviter qu'une prochaine loi ne «déqualifie» la société et n'organise la privatisation de son capital.

L'on ne peut dès lors s'empêcher de faire un parallèle avec la décision $\mathrm{n}^{\circ}$ 2004-501 DC, dans laquelle le Conseil déclarait que «le législateur a confirmé $1[a]$ qualité de services publics nationaux [de EDF et de GDF] ; qu'il a garanti, conformément au neuvième alinéa du préambule de la Constitution de 1946, la participation majoritaire de l'État [...] dans le capital de ces sociétés; que l'abandon de cette participation majoritaire ne pourrait résulter que d'une loi ultérieure ${ }^{91}$, loi de transfert au secteur privé intervenue le 7 décembre $2006^{92}$.

\section{L'organisation du suffrage}

L'organisation du suffrage conditionne directement l'expression de la volonté générale. Aussi, le Conseil constitutionnel ne manque-t-il pas de rappeler qu'il ne «dispose pas d'un pouvoir général d'appréciation et de décision de même nature que celui du Parlement ${ }^{93}$ et qu'il opère un contrôle minimum ${ }^{94}$.

\section{La clarté et la loyauté du scrutin}

En vue de l'instauration des conseillers territoriaux - susceptibles d'être élus pour la première fois en mars 2014 -, le législateur procède à la réduction des mandats des conseillers généraux et régionaux afin qu'ils échoient en mars 2014. Or, la modification desdits mandats ne peut être justifiée que par un intérêt général hypothétique sauf à considérer que le législateur futur soit contraint de procéder à la création des conseillers territoriaux. Cependant, le Conseil, dans la décision Renouvellement des conseils généraux et régionaux ${ }^{95}$, conforte la démarche anticipée du législateur et considère que celle-ci s'inscrit dans le respect des exigences de clarté et de loyauté des élections des conseillers régionaux et généraux de 2010 et 2011. Selon les juges, il est préférable de prévoir la concomitance du renouvellement des mandats généraux et régionaux en vue de l'entrée en vigueur d'une réforme, même éventuelle, que de devoir prolonger ou raccourcir des mandats en cours, une fois la réforme adoptée ${ }^{96}$. Afin d'appuyer sa position, le Conseil ajoute que la modification du calendrier peut être justifiée, indépendamment de toute réforme ultérieure, par l'objectif de favoriser une plus forte participation du corps électoral à chacune des consultations concernées dès lors qu'elles seraient concomitantes ${ }^{97}$.

\section{L'égalité devant le suffrage}

Concernant l'actualisation de la répartition des sièges et de la délimitation des circonscriptions pour l'élection des députés, les requérants contestent la méthode dite des tranches utilisée par le Gouvernement au motif qu'une autre méthode permet une meilleure répartition des sièges sur des bases démographiques ${ }^{98}$. Le Conseil, dans la décision $\mathrm{n}^{\circ}$ 2010-602 DC du 18 février 2010, Loi ratifiant l'ordonnance $n^{\circ}$ 2009-935 du 29 juillet 2009 portant répartition des sièges et délimitation des circonscriptions pour l'élection des députés, rejette l'ensemble des griefs soulevés pour deux raisons. D'une part, cette méthode a été approuvée par une commission indépendante prévue à l'article $25 \mathrm{de}$ la Constitution. Et les juges de rappeler que l'utilisation d'une méthode plus égalitaire - que le Conseil récuse " aurait conduit à augmenter sensiblement le nombre de départements n'élisant qu'un seul député ${ }^{99}$. Or, dans une décision du 8 janvier 2009, la rue de Montpensier affirmait que le maintien d'un minimum de deux députés pour chaque département n'était plus «justifié par un impératif d'intérêt général susceptible d'atténuer la portée de la règle fondamentale selon laquelle l'Assemblée nationale doit être élue sur des bases essentiellement

90. CC, déc. $\mathrm{n}^{\circ}$ 2010-601 DC du 4 février 2010, Loi relative à l'entreprise publique La Poste et aux activités postales, cons. 4. Le Conseil relève que le législateur a précisé que «cette transformation ne peut avoir pour conséquence de remettre en cause le caractère de service public national de La Poste» et que «le capital de la société est détenu par l'État, actionnaire majoritaire, et par d'autres personnes morales de droit public» (cons. 2).

91. CC, déc. $\mathrm{n}^{\circ}$ 2004-501 DC du 5 août 2004, Loi relative au service public de l'électricité et du gaz et aux entreprises électriques et gazières, cons. 14 .

92. Loi $n^{\circ}$ 2006-1537 du 7 décembre 2006 relative au secteur de l'énergie, parue au JORF, $\mathrm{n}^{\circ}$ 284, 8 décembre 2006. Sur ce point, voir G. Marcou, «Que reste-t-il de l'alinéa 9 du préambule de la Constitution de 1946? ", AJDA, 2007, p. 192-198.

93. CC, déc. Renouvellement des conseils généraux et régionaux, cons. 13 ; CC, déc. $\mathrm{n}^{\circ} 2010-602$ DC du 18 février 2010 , Loi ratifiant l'ordonnance $n^{\circ}$ 2009-935 du 29 juillet 2009 portant répartition des sièges et délimitation des circonscriptions pour l'élection des députés, cons. 20.

94. CC, déc. Collectivités territoriales, cons. 41.

95. CC, déc. Renouvellement des conseils généraux et régionaux.

96. Ibid., cons. 14

97. Ibid.

98. CC, déc. $\mathrm{n}^{\circ}$ 2010-602 DC du 18 février 2010, Loi ratifiant l’ordonnance $n^{\circ} 2009-935$ du 29 juillet 2009 portant répartition des sièges et délimitation des circonscriptions pour l'élection des députés, cons. 11.

99. Ibid., cons. 14 
démographiques ${ }^{100}$. D'un côté, le minimum de deux députés n'est donc plus un impératif mais il demeure, de l'autre, un motif pour écarter une méthode pourtant plus égalitaire. D'autre part, le Conseil constate que les inégalités devant le suffrage ont été réduites de manière importante et que donc l'ordonnance ratifiée réalise un progrès par rapport à la situation antérieure ${ }^{101}$. Si sa jurisprudence n'apparaît donc pas tout à fait établie, le juge confirme, dans cette décision, sa prudence et le caractère restreint ${ }^{102}$ de son contrôle.

En revanche, dans la décision Collectivités territoriales, le Conseil affine sa jurisprudence. L'ampleur de la réforme lui permet d'être plus exigeant ${ }^{103}$ dans la mesure où elle entraîne un redécoupage de l'ensemble des circonscriptions et non une simple actualisation que le Conseil aurait validée au seul motif qu'elle constituerait un progrès par rapport à la situation antérieure. Tout d'abord, le Conseil accepte le seuil minimal de quinze conseillers territoriaux par département mais précise que les départements concernés par ce minimum doivent être exclus pour le calcul de la moyenne de référence du nombre de conseillers par région ${ }^{104}$. Ensuite, le Conseil, intransigeant et précis, censure la répartition des sièges dans six départements où l'écart entre le ratio du nombre de conseillers territoriaux par rapport à la population départementale et la moyenne régionale dépasse $20 \%{ }^{105}$. Enfin, constatant que les régions visées par la censure ne sont pas séparables du reste de la distribution des sièges, il invalide l'ensemble du tableau de répartition des sièges de conseillers territoriaux ${ }^{106}$.

100. CC, déc. $\mathrm{n}^{\circ}$ 2008-573 DC du 8 janvier 2009, Loi relative à la commission prévue à l'article 25 de la Constitution et à l'élection des députés, cons. 23 ; voir également le commentaire de la décision $\mathrm{n}^{\circ}$ 2010-602 DC du 18 février 2010, Les Cahiers du Conseil constitutionnel, cahier $\mathrm{n}^{\circ} 28,2010, \mathrm{p} .14$.

101. CC, déc. $\mathrm{n}^{\circ}$ 2010-602 DC du 18 février 2010, Loi ratifiant l’ordonnance $n^{\circ}$ 2009-935 du 29 juillet 2009 portant répartition des sièges et délimitation des circonscriptions pour l'élection des députés, cons. 15.

102. Commentaire de la décision $\mathrm{n}^{\circ}$ 2010-602 DC du 18 février 2010, Les Cahiers du Conseil constitutionnel, cahier $\mathrm{n}^{\circ}$ 28, $2010, \mathrm{p} .18$.

103. Commentaire de la décision $\mathrm{n}^{\circ}$ 2010-618 DC du 9 décembre 2010, Les Nouveaux Cahiers du Conseil constitutionnel, cahier $\mathrm{n}^{\circ} 30,2010, \mathrm{p} .20$.

104. CC, déc. Collectivités territoriales, cons. 39.

105. Ibid., cons. 41.

106. Ibid. 